\title{
Numerical Simulation of the Characteristics of Electrons in Bar-plate DC Negative Corona Discharge Based on a Plasma Chemical Model
}

\author{
Kang-Lin Liu ${ }^{\dagger}$, Rui-Jin Liao* and Xue-Tong Zhao*
}

\begin{abstract}
In order to explore the characteristics of electrons in DC negative corona discharge, an improved plasma chemical model is presented for the simulation of bar-plate DC corona discharge in dry air. The model is based on plasma hydrodynamics and chemical models in which 12 species are considered. In addition, the photoionization and secondary electron emission effect are also incorporated within the model as well. Based on this model, electron mean energy distribution (EMED), electron density distribution (EDD), generation and dissipation rates of electron at 6 typical time points during a pulse are discussed emphatically. The obtained results show that, the maximum of electron mean energy (EME) appears in field ionization layer which moves towards the anode as time progresses, and its value decreases gradually. Within a pulse process, the electron density (ED) in cathode sheath almost keeps 0 , and the maximum of ED appears in the outer layer of the cathode sheath. Among all reactions, R1 and R2 are regarded as the main process of electron proliferation, and $\mathrm{R}_{22}$ plays a dominant role in the dissipation process of electron. The obtained results will provide valuable insights to the physical mechanism of negative corona discharge in air.
\end{abstract}

Keywords: Corona discharge, Plasma chemical, Numerical simulation

\section{Nomenclature}

$n_{e} \quad$ electron density

$N^{+} \quad$ positive ion density

$N^{-} \quad$ negative ion density

$\mathrm{N}_{\mathrm{n}} \quad$ neutral particle density

$N_{i} \quad$ particles number of species $i$,

$\Delta \varepsilon_{k}^{e} \quad$ energy lost per-electron in the reaction $k$

$M_{e} \quad$ electron mobility

$M_{\varepsilon} \quad$ energy mobility

$D_{e} \quad$ electron diffusion coefficient

$D_{\varepsilon} \quad$ energy diffusion coefficients

$\Phi \quad$ electrostatic potential

$\varepsilon \quad$ electron mean energy

$E \quad$ unit charge $(1.6021892 \times 10-19 \mathrm{c})$

$K$ total number of reactions

$K_{b} \quad$ boltzmann constant

$T$ environment temperature

$E \quad$ air permittivity

$P \quad$ gas pressure

$R \quad$ horizontal coordinate

$Z \quad$ vertical coordinate

$P_{q} \quad$ quenching pressure for the photo-ionization states

$R_{e} \quad$ rate of electron generation/dissipation

$R_{k} \quad$ rate of progress of reaction $k$ with electron

$\dagger$ Corresponding Author: State Key Laboratory of Power Transmission Equipment and System Security and New Technology, Chongqing University, China. (cquklliu@cqu.edu.cn)

* State Key Laboratory of Power Transmission Equipment and System Security and New Technology, Chongqing University, China. (cquklliu@cqu.edu.cn)

Received: December 1, 2014; Accepted: March 31, 2015 participation

$R_{\text {ion }}$ rate of ionization

$R_{p h} \quad$ rate of photoionization

$R_{r e c}^{e i}$ rate of electron-ion

$R_{\text {rec }}^{i i^{\prime}}$ rate of ion-ion recombination

$R_{\text {att }} \quad$ rate of electron adsorption

$\Gamma_{e} \quad$ secondary emission coefficient

$\Gamma_{i} \quad$ surface interaction coefficient

$M_{i} \quad$ ion mass of species $i$

$V_{\text {e.th }}$ electron thermal velocity

$\Gamma_{e} \quad$ electron density flux

$\Gamma_{\varepsilon} \quad$ electron energy flux

$\Gamma_{i} \quad$ flux density of species $i$

$E \quad$ electrical field

$N$ boundary normal vector

\section{Introduction}

As a typically non-thermal discharge, corona discharge is usually generated on sharp points, edges or thin wires where the electric field is strongly concentrated $[1,2]$. With the rapid development of extra high-voltage (EHV) transmission lines, corona discharge in air has become one of the critical problems associated with high-voltage transmission lines. In many occasion, especially in electric transmission, corona discharge is undesirable. For example, many chemical reactions happen along with corona discharge, and this results in the production of many hazardous substance with great corrosivity and oxidizability, such as ozone and nitrogen oxides. And corona discharge will 
produce high frequency pulse currents which contain higher harmonic. This will bring in interference and affect wireless communications. What's worse, corona discharge can cause energy losses. The higher the voltage grade, the more energy loss $[3,4]$. In the meantime, corona discharge has been employed as an effective tool in many industrial applications such as electrostatic precipitators, disinfection, ozone generators, surface treatment, ignition, electrostatic painting, and so on [5-7]. In recent year, the application of corona discharge has been expanded broadly. Kinetic analysis has been developed for pollution control using none thermal plasma by Penetrante [8]. Masuda used surface-induced plasma chemical process for the destruction of gaseous pollutants [9]. Corona discharge is also used for gas separation [10,11]. What's more, Deli [12] carried out experiments on the cleaning of air using DC streamer discharge. In the past decades, great focuses have been paid on the mechanism of corona discharge in air [13]. As has been reported, the collisions between the highenergy electrons and the neutral molecules are the major ionization processes of air corona discharge. Meanwhile, electrons are the main energy transfer carrier between electric field and heavy particles [14]. Consequently, investigation on characteristics of electrons in corona discharge during a pulse cycle can be of important value to understand the physical mechanism of air discharge, and further provide valuable references to the suppression of corona discharge of transmission lines [15].

A variety particles such as excited-state species, ions, free radicals, are generated during the microcosmic process of corona discharge, which would lead to complex physical and chemical reactions. In addition, it will also determine the multiformity of interaction between them. The preferable approach to carry out in-depth investigations within the microcosmic process of corona discharge should be numerical modeling because of the lack of effective measurement methods in plasma discharge process [16]. Chemical reactions and non-equilibrium can better reflect the microcosmic process of gas discharge, which should be incorporated within the discharge simulation, and it will provide more important information to gas discharge investigation. Nahomy et al. [17] presented a comparatively comprehensive fusion of 430 chemistry reactions in air discharge with the demerit of large-scale computation. A two-dimensional physical model proposed by Pancheshnyi et al. [18] for discharge in $\mathrm{N}_{2}-\mathrm{O}_{2}(9: 1)$ mixed gas under 760 Torr was improved based on the selection of 10 kinds of primary particles from Nahomy's model, which was further employed to analyze electric field distribution, densities of charged particles, reaction rates and other discharge parameters, and the obtained results fairly correspond to the experimental data. Liu et al. $[19,20]$ established a fluid-chemical mixed kinetic model for corona discharge under atmospheric pressure and the computed V-I curve and electron temperature curve accorded well with the experiment values carried by
Antao [21]. Based on this model, current density, electron density, and positive/negative ion densities distribution are discussed. Furthermore, collision reactions were added into fluid dynamics model, and an improved model for negative corona discharge was obtained to acquire the information of electric field distribution, rates of collision reaction, development tendency of space charge, electron density distribution and other characteristics in the microcosmic process of Trichel pulse [22]. To sum up, characteristics of electrons were roughly analyzed by the presented simulation models, and until now no specific models are proposed for analysis of characteristics of electrons during the microcosmic process of corona discharge.

This paper presents a new plasma hybrid model for the simulation of DC negative corona discharge under atmospheric air. Using the proposed novel simulation model, this work aims at discussing some microcosmic characteristics such as electron mean energy distribution, electron density distribution, generation and dissipation performances of electrons along the axis during a pulse cycle in order to understand the main physical mechanisms. The rest of the present paper is organized as follows. The detailed descriptions are presented for the negative corona discharge model in Sec. 2. Sec. 3 presents the corresponding results and discussions. Finally, the conclusions are summarized in Sec. 4.

\section{Mathematical Model}

In substance, the solution of the microcosmic mechanism for corona discharge is to convert the electron conservation equation, the electron energy conservation, the heavy species multi-component diffusion transport equation, governing equations for heavy particles, and the Poisson's equation into the appropriate system of partial differential equations which are then normalized and solved by means of discrete numerical difference equations.

\subsection{Governing equations} [23]:

The balance governing equation for electron density is

$$
\frac{\partial n_{e}}{\partial t}+\nabla \cdot \boldsymbol{\Gamma}_{e}=R_{e}
$$

$R_{e}$ is related to ionization or recombination, photoionization processes and secondary emission processes on the cathode and $\Gamma_{e}$ is expressed as:

$$
\Gamma_{e}=-\nabla\left(D_{e} n_{e}\right)+\mu_{e} n_{e} \nabla \varphi
$$

The electron mean energy density equation is given as [24]: 


$$
\frac{\partial}{\partial t}\left(n_{e} \boldsymbol{\varepsilon}\right)+\nabla \cdot \boldsymbol{\Gamma}_{\varepsilon}=-e \boldsymbol{\Gamma}_{e} \cdot \nabla \varphi+e \sum_{k=1}^{K} \Delta \varepsilon_{k}^{e} R_{k}
$$

$\Gamma_{\varepsilon}$ is described by the following equation:

$$
\Gamma_{\varepsilon}=n_{e} \varepsilon \mu_{\varepsilon} \nabla \varphi-D_{\varepsilon} \nabla n_{e} \varepsilon
$$

These energy transport coefficients are given by [19]:

$$
\mu_{\varepsilon}=\frac{5 \mu_{e}}{3}, \quad D_{\varepsilon}=\frac{5 D_{e}}{3}
$$

These transport coefficients of electrons are obtained from solving the Boltzmann equation [25].The streamer discharge in air in highly overstressed gap is very fast (several nanoseconds). On the several nanoseconds time scale, the diffusion and mobility phenomenon of heavy species (ions and neutral particles) have almost no influence on the discharge propagation. So the diffusion and mobility coefficients of heavy species can be ignored [18]. The governing equations for ions and neutral particles are given by

$$
\left\{\begin{array}{l}
\frac{\partial n_{i}^{+}}{\partial t}=R_{i o n}+R_{p h}-R_{r e c}^{e i}-R_{r e c}^{i i} \\
\frac{\partial n_{i}^{-}}{\partial t}=R_{a t t}-R_{r e c}^{i i} \\
\frac{\partial n_{n}}{\partial t}=R_{r e c}^{e i}+R_{r e c}^{i i}-R_{i o n}-R_{p h}-R_{a t t}
\end{array}\right.
$$

Here, subscript $i$ indicates species of ion $\left(\mathrm{O}_{2}^{+}, \mathrm{O}_{4}{ }^{+}, \mathrm{O}_{2}^{-}\right.$, $\mathrm{O}^{-}, \mathrm{N}_{2}{ }^{+}, \mathrm{N}_{4}^{+}$, and $\mathrm{N}_{2} \mathrm{O}_{2}{ }^{+}$.

The electric field in the discharge gap is calculated using Poisson's equation [18]:

$$
\varepsilon \nabla^{2} \varphi=-e\left(\sum n_{i}^{+}-\sum n_{i}^{-}-n_{e}\right)
$$

\subsection{Air chemistry and transport}

The essence of air discharge is regarded as collision between charged particles and neutral air molecules (atoms), molecules (atoms) clusters and other basic particles, as well as results of the collision between these particles and electrode surfaces [19]. The reaction pathways are adopted primarily from the mechanism of Pancheshnyi [18]. Table 1 lists the reaction processes that are taken into account in the model [26, 27].

The charged ions are quenched to the neutral species due

\begin{tabular}{|c|c|c|c|}
\hline No. & Reaction & Rate coefficient & \\
\hline $\mathrm{R}_{1}$ & $\mathrm{~N}_{2}+\mathrm{e} \rightarrow 2 \mathrm{e}+\mathrm{N}_{2}^{+}$ & $f(\varepsilon)$ & 15.6 \\
\hline $\mathrm{R}_{2}$ & $\mathrm{O}_{2}+\mathrm{e} \rightarrow 2 \mathrm{e}+\mathrm{O}_{2}^{+}$ & $F(\varepsilon)$ & 12.06 \\
\hline $\mathrm{R}_{3}$ & $\mathrm{~N}_{2}^{+}+2 \mathrm{~N}_{2} \rightarrow \mathrm{N}_{4}^{+}+\mathrm{N}_{2}$ & $5.0 \times 10^{-41}$ & \\
\hline $\mathrm{R}_{4}$ & $\mathrm{~N}_{2}^{+}+\mathrm{N}_{2}+\mathrm{O}_{2} \rightarrow \mathrm{N}_{4}^{+}+\mathrm{O}_{2}$ & $5.0 \times 10^{-41}$ & \\
\hline $\mathrm{R}_{5}$ & $\mathrm{~N}_{4}^{+}+\mathrm{O}_{2} \rightarrow \mathrm{O}_{2}^{+}+2 \mathrm{~N}_{2}$ & $2.5 \times 10^{-16}$ & \\
\hline $\mathrm{R}_{6}$ & $\mathrm{~N}_{2}^{+}+\mathrm{O}_{2} \rightarrow \mathrm{O}_{2}^{+}+\mathrm{N}_{2}$ & $1.04 \times 10^{-15} T^{-0.5}$ & \\
\hline $\mathrm{R}_{7}$ & $2 \mathrm{~N}_{2}+\mathrm{O}_{2}^{+} \rightarrow \mathrm{N}_{2} \mathrm{O}_{2}^{+}+\mathrm{N}_{2}$ & $8.1 \times 10^{-38} T^{-2}$ & \\
\hline $\mathrm{R}_{8}$ & $\mathrm{~N}_{2} \mathrm{O}_{2}^{+}+\mathrm{N}_{2} \rightarrow \mathrm{O}_{2}^{+}+2 \mathrm{~N}_{2}$ & $14.6 T^{5.3} \exp (-2357 / T)$ & \\
\hline $\mathrm{R}_{9}$ & $\mathrm{~N}_{2} \mathrm{O}_{2}^{+}+\mathrm{O}_{2} \rightarrow \mathrm{O}_{4}^{+}+\mathrm{N}_{2}$ & $1.0 \times 10^{-15}$ & \\
\hline $\mathrm{R}_{10}$ & $\mathrm{O}_{2}^{+}+\mathrm{O}_{2}+\mathrm{N}_{2} \rightarrow \mathrm{O}_{4}^{+}+\mathrm{N}_{2}$ & $2.04 \times 10^{-34} T^{-3.2}$ & \\
\hline $\mathrm{R}_{11}$ & $\mathrm{O}_{2}^{+}+2 \mathrm{O}_{2} \rightarrow \mathrm{O}_{4}^{+}+\mathrm{O}_{2}$ & $2.04 \times 10^{-34} T^{-3.2}$ & \\
\hline $\mathrm{R}_{12}$ & $\mathrm{O}_{4}^{+}+\mathrm{e} \rightarrow 2 \mathrm{O}_{2}$ & $1.4 \times 10^{-12}\left(300 / T_{e}\right)^{0.5}$ & \\
\hline $\mathrm{R}_{13}$ & $\mathrm{O}_{2}^{+}+\mathrm{e} \rightarrow 2 \mathrm{O}$ & $2.42 \times 10^{-13}\left(300 / T_{e}\right)$ & \\
\hline $\mathrm{R}_{14}$ & $2 \mathrm{O}_{2}+\mathrm{e} \rightarrow \mathrm{O}_{2}+\mathrm{O}_{2}^{-}$ & $2.0 \times 10^{-41}\left(300 / T_{e}\right)$ & \\
\hline $\mathrm{R}_{15}$ & $\mathrm{O}_{4}^{+}+\mathrm{O}_{2^{-}} \rightarrow 3 \mathrm{O}_{2}$ & $1.0 \times 10^{-13}$ & \\
\hline $\mathrm{R}_{16}$ & $\mathrm{O}_{4}^{+}+\mathrm{O}_{2}^{-}+\mathrm{N}_{2} \rightarrow 3 \mathrm{O}_{2}+\mathrm{N}_{2}$ & $2.0 \times 10^{-37}$ & \\
\hline $\mathrm{R}_{17}$ & $\mathrm{O}_{4}^{+}+\mathrm{O}_{2}^{-}+\mathrm{O}_{2} \rightarrow 3 \mathrm{O}_{2}+\mathrm{O}_{2}$ & $2.0 \times 10^{-37}$ & \\
\hline $\mathrm{R}_{18}$ & $\mathrm{O}_{2}^{+}+\mathrm{O}_{2^{-}}+\mathrm{N}_{2} \rightarrow 2 \mathrm{O}_{2}+\mathrm{N}_{2}$ & $2.0 \times 10^{-37}$ & \\
\hline $\mathrm{R}_{19}$ & $\mathrm{O}_{2}^{+}+\mathrm{O}_{2}^{-}+\mathrm{O}_{2} \rightarrow 3 \mathrm{O}_{2}$ & $2.0 \times 10^{-37}$ & \\
\hline $\mathrm{R}_{20}$ & $\mathrm{O}+\mathrm{O}_{2}+\mathrm{N}_{2} \rightarrow \mathrm{O}_{3}+\mathrm{N}_{2}$ & $2.5 \times 10^{-46}$ & \\
\hline $\mathrm{R}_{21}$ & $\mathrm{O}+2 \mathrm{O}_{2} \rightarrow \mathrm{O}_{3}+\mathrm{O}_{2}$ & $2.5 \times 10^{-46}$ & \\
\hline $\mathrm{R}_{22}$ & $\mathrm{e}+\mathrm{N}_{2}^{+}+\mathrm{N}_{2} \rightarrow 2 \mathrm{~N}_{2}$ & $6.07 \times 10^{-34} T_{e}^{-2.5}$ & \\
\hline $\mathrm{R}_{23}$ & $2 \mathrm{e}+\mathrm{N}_{2}^{+} \rightarrow \mathrm{N}_{2}+\mathrm{e}$ & $5.651 \times 10^{-27} T_{e}^{-0.8}$ & \\
\hline $\mathrm{R}_{24}$ & $\mathrm{O}_{2}^{+}+\mathrm{O}^{-} \rightarrow \mathrm{O}+\mathrm{O}_{2}$ & $3.46 \times 10^{-12} T_{e}^{-0.5}$ & \\
\hline $\mathrm{R}_{25}$ & $\mathrm{~N}_{2}+\mathrm{e} \rightarrow \mathrm{e}+\mathrm{N}_{2}$ & $f(\varepsilon)$ & 1.0 \\
\hline $\mathrm{R}_{26}$ & $\mathrm{O}_{2}+\mathrm{e} \rightarrow \mathrm{e}+\mathrm{O}_{2}$ & $F(\varepsilon)$ & 1.0 \\
\hline $\mathrm{R}_{27}$ & $\mathrm{O}_{2}+\mathrm{e} \rightarrow \mathrm{O}+\mathrm{O}^{-}$ & $f(\varepsilon)$ & 3.6 \\
\hline
\end{tabular}
to the surface reactions:

$$
\begin{gathered}
N_{2}^{+} \rightarrow N_{2}, N_{4}^{+} \rightarrow 2 N_{2}, O_{2}^{+} \rightarrow O_{2}, \\
O_{4}^{+} \rightarrow 2 O_{2}, O_{2}^{+} N_{2} \rightarrow N_{2}+O_{2}, O_{2}^{-} \rightarrow O_{2}
\end{gathered}
$$

Table 1. Kinetic processes considered in the model

Photoionization is one of the main factors promoting discharge propagation in air [18, 28-31]. Recently, the detailed studies on photoionization were given through the comparison of experiments and simulations by Nijdam and Wormeester [30]. Photoionization of corona discharge in air is caused by the radiation in the wavelength range 980-1025 tenthmeter resulting from the radiative transitions from the three single states of $\mathrm{N}_{2}\left(b^{1} \prod_{u}, b^{\prime 1} \sum_{u}^{+}\right.$and $\left.c_{4}^{\prime 1} \sum_{u}^{+}\right)$to the ground state $\mathrm{N}_{2}\left(\mathrm{x}^{1} \sum_{g}^{+}\right)$[32]. Based on the above assumptions, Zheleznyak [31] proposes the rate $R_{p h}$ of a non-local photoionization, as expressed in the following equations:

$$
\begin{gathered}
N_{2}\left(b^{1} \prod_{u}, b^{\prime 1} \sum_{u}^{+}, c_{4}^{\prime 1} \sum_{u}^{+}\right) \rightarrow N_{2}\left(x^{1} \sum_{g}^{+}\right)+h v \\
\mathrm{O}^{2}+h v \rightarrow \mathrm{O}_{2}^{+}+e
\end{gathered}
$$

Here the Eddington approximation method [33] is used to calculate $R_{\mathrm{ph}}$ :

$$
R_{\mathrm{ph}}=\frac{1}{4 \pi} \cdot \frac{p_{\mathrm{q}}}{p+p_{\mathrm{q}}} \int_{V} d^{3} \boldsymbol{r}_{1} \frac{R_{\mathrm{ion}}\left(\boldsymbol{r}_{1}\right)}{\left|\boldsymbol{r}-\boldsymbol{r}_{1}\right|^{2}} h\left(\left|\boldsymbol{r}-\boldsymbol{r}_{1}\right| \cdot p\right)
$$

where $1 / 4 \pi$ is the normalizing constant, $r$ is the observation point, $r_{1}$ is the source point, and $h\left(\left|\boldsymbol{r}-\boldsymbol{r}_{1}\right| \cdot p\right)$ is the coefficient of absorption of the ionizing radiation in the 
medium.

\subsection{Boundary conditions and computational imple- mentation}

Fig. 1(a) gives the description of the schematic diagram of bar-plate DC negative corona discharge in air at atmospheric environment. A copper bar, with a tip radius curvature $R(0.4 \mathrm{~mm})$ and length $L(2 \mathrm{~mm})$, is perpendicular to an infinitely large plate which is made of copper and has a radius of $1 \mathrm{~cm}$ at a distance D $(3.3 \mathrm{~mm})$. Negative potential is applied to the bar, the plane electrode is grounded as zero potential, and the zero charge boundary conditions [34] $(\boldsymbol{n} \cdot(\varepsilon \nabla \varphi)=0)$ are used at the open boundary. The ambient gas is air $\left(\mathrm{O}_{2}: \mathrm{N}_{2}=1: 4\right)$ at room temperature $(300 \mathrm{~K})$ and atmospheric pressure $(1.0 \mathrm{~atm})$. Within numerical model, the bar-plate electrode system model is simplified into a two-dimensional representation using the rotational symmetry. The computational domain is highlighted with green in Fig. 1(a). Some important boundary conditions are given as follows.

The electron flux at the electrode is expressed as [35]

$$
\Gamma_{e} \cdot \boldsymbol{n}=\frac{1-\gamma_{e}}{1+\gamma_{e}}\left[-\left(2 \alpha_{e}-1\right) \mu_{e} \boldsymbol{E} \cdot \boldsymbol{n} n_{e}+\frac{1}{2} v_{e, t h} n_{e}\right]
$$

$a_{e}$ is 1 when the electron flux is directed toward the electrode, and zero otherwise. The electron energy flux at the cathode and the anode is:

$$
\Gamma_{e} \cdot \boldsymbol{n}=\frac{1}{3} v_{e, t h} \boldsymbol{\varepsilon} n_{e}
$$

$v_{e, t h}=\sqrt{8 k_{B} T / \pi m_{e}}$ is the electron thermal velocity. At the electrode surfaces, the ions and unstable neutral particles are quenched to stable neutral particles due to surface reactions (Eq. (8)).

The ion fluxes at the electrode are expressed as follows [34]:

$$
\boldsymbol{\Gamma}_{\boldsymbol{i}} \cdot \boldsymbol{n}=\frac{\gamma_{i}}{4} \sqrt{\frac{8 k_{B} T}{\pi m_{i}}} n_{i}
$$

$\gamma_{i}$ specifies the probability that species $i$ will react at the surface, and The normal gradient of the density of all species is zero $\left[\boldsymbol{n} \cdot\left(\nabla n_{i}\right)=0\right]$ at the open boundaries.

A Gaussian distribution of seed electrons and positive ions with a maximum value of $1016 \mathrm{~m}^{-3}$ and width of 25 $\mu \mathrm{m}$ is set at the needle tip according to the following equation [25]:

$$
\left.\sum_{i} n_{i}^{+}(r, z)\right|_{t=0}=\left.n_{e}\right|_{t=0}=N_{\max } \times \exp \left[-\frac{\left(r-r_{0}\right)^{2}}{2 s_{0}^{2}}-\frac{\left(z-z_{0}\right)^{2}}{2 s_{0}^{2}}\right]
$$

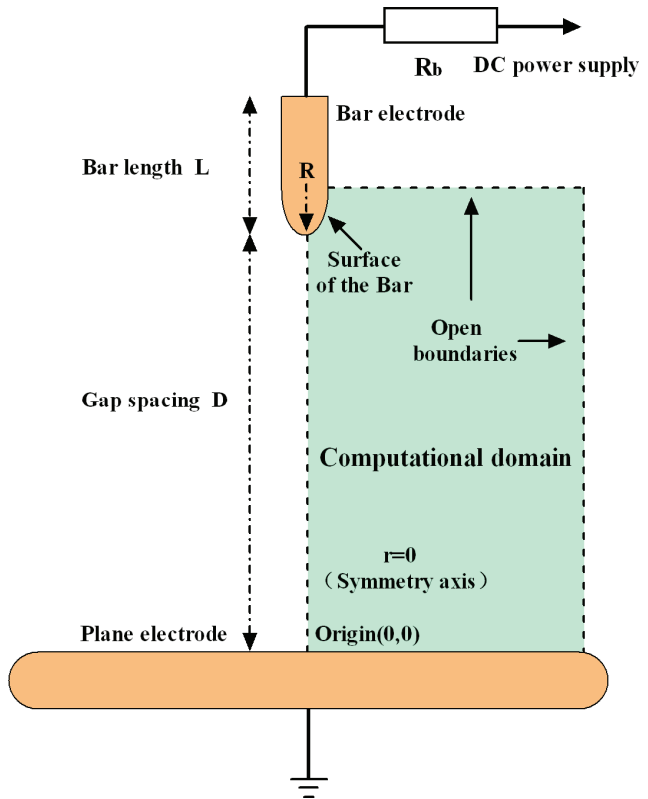

(a)

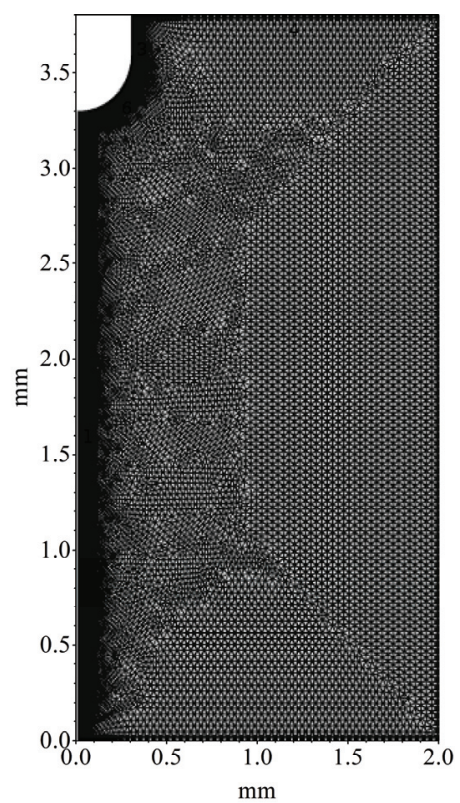

(b)

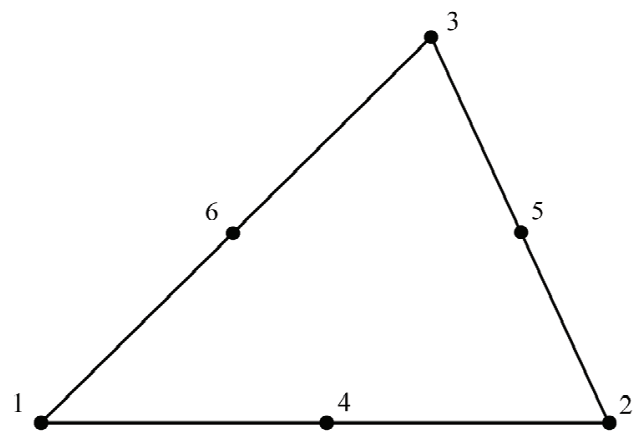

(c)

Fig. 1. Schematic of the computational model: (a) schematic diagram of the negative corona discharge; (b) mesh figure; (c) triangle of six-node second order 


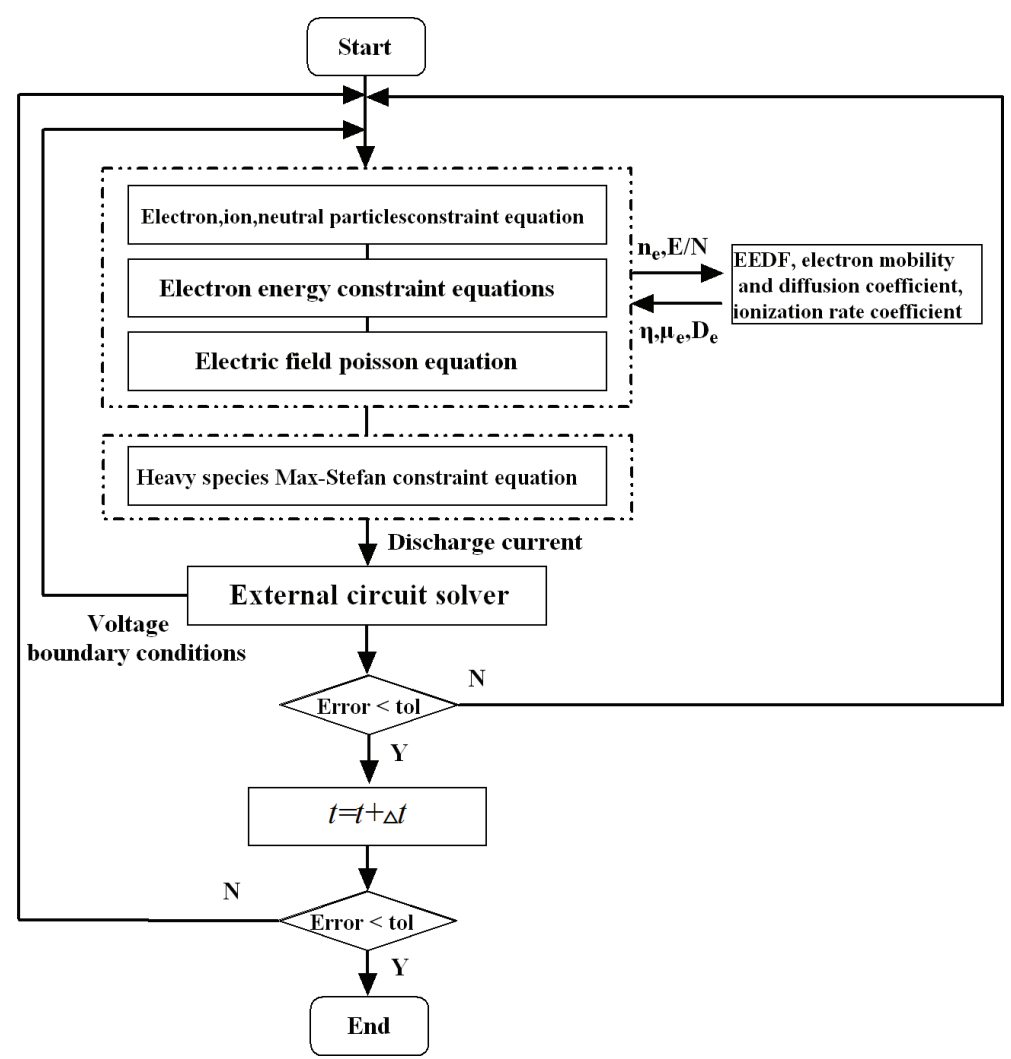

Fig. 2. Flowchart of computer program

where $N_{\max }=10^{16} \mathrm{~m}^{-3}, r_{0}=0 \mu \mathrm{m}, \mathrm{z}_{0}$ is the needle tip, $s_{0}=$ $25 \mu \mathrm{m} .\left.\sum_{i} n_{i}^{+}(r, z)\right|_{t=0}$ (The subscript $i$ denoting $\mathrm{O}_{2}{ }^{+}, \mathrm{O}_{4}{ }^{+}$, $\mathrm{N}_{2}^{+}, \mathrm{N}_{4}^{+}$, and $\mathrm{N}_{2} \mathrm{O}_{2}^{+}$), and $\left.n_{e}\right|_{t=0}$ are positive ion and electron density at $\mathrm{t}=0$. The initial condition has been confirmed to only speed up the pulse formation and not to change the discharge characteristics $[25,36]$.

The model in this work is implemented in COMSOL Multiphysic package based on finite element method. Fully implicit time stepping method is used for the Eqs. (1), (3), (6) and (7). It has been proved that the corona discharge propagates along the axial direction, therefore, due to the high electric field and charge densities [37], a very fine 'unstructured mesh (Fig. 1(b))' need to be used along the axial symmetry line [38]. Triangle of six-node second order (Fig. 1 (c)) is used in the mesh, and the mesh parameters are shown in Table 2. Finally, the flowchart of the computer program is shown in Fig. 2.

Table 2. Mesh statistics

\begin{tabular}{c|c}
\hline \multicolumn{2}{c}{ Mesh Parameter } \\
\hline Triangular elements & 22218 \\
\hline Edge elements & 608 \\
\hline Minimum element quality & 0.7527 \\
\hline Average element quality & 0.9785 \\
\hline Element area ratio & 0.03552 \\
\hline Mesh area & $7.469 \times 10^{-6} \mathrm{~m}^{2}$ \\
\hline Elements nodal points & 6 \\
\hline
\end{tabular}

\section{Results and Discussion}

\subsection{Individual pulse waveform}

In this paper, 6 typical time points $\left(\mathrm{t}_{1}-\mathrm{t}_{6}\right)$ is used to describe microcosmic characteristics of electrons during a pulse cycle in bar-plate DC negative corona discharge. Fig. 3 shows the pulse current waveform in the electrode gap where the negative DC voltage applied to the bar is $5.0 \mathrm{kV}$. The pulse of the discharge current is characterized by a fast rising front (as short as $1.6 \mathrm{ns)}$ ) and a slow decaying (about $60 \mathrm{~ns}$ ), which has been confirmed by Zentner [39] and Soria [40]'s experiment. The maximum discharge current rapidly increases to $0.195 \mathrm{~A}$ from $\mathrm{t}_{1}-\mathrm{t}_{2}$. Then, it starts to

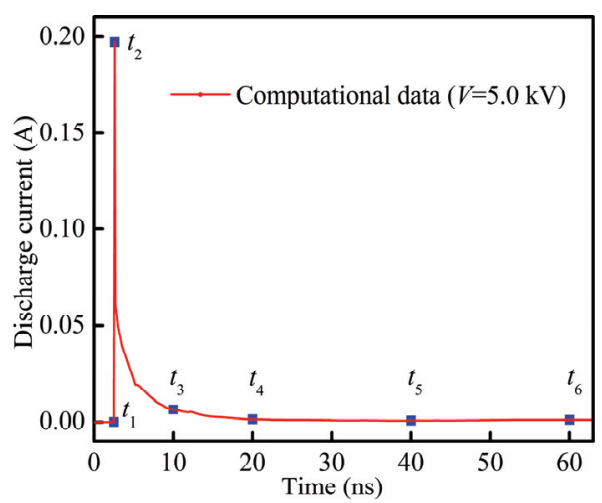

Fig. 3. The discharge current waveform in the electrode gap 


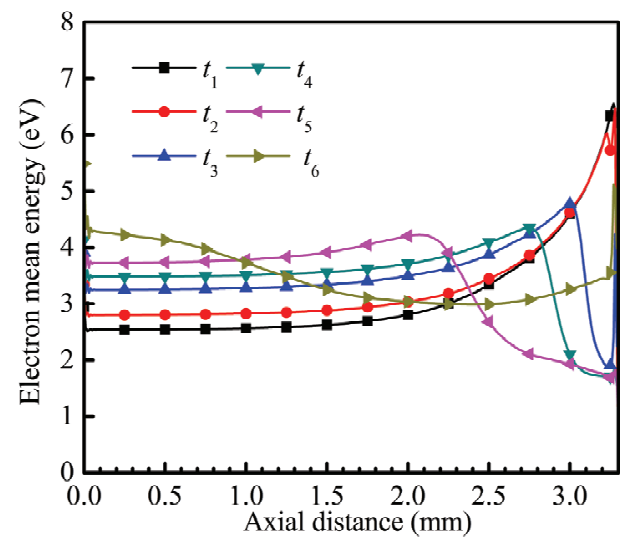

Fig. 4. Electron mean energy distribution along the axis

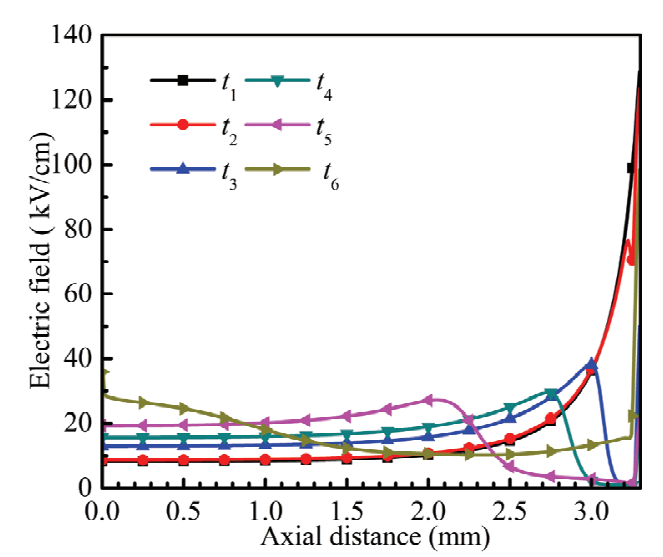

Fig. 5. Electric field distribution along the axis

decrease slowly from $t_{2}-t_{4}$. The first peak is generated due to the field ionization. During $t_{1}$ and $t_{2}$, the electric field keeps at a high level as shown in Fig. 5. A large electrons are produced in the cathode for the impact of positive ion in the cathode and field ionization. Electrons are accelerating in the electric field, and the number of electron and positive ion are growing exponentially because of ionization. The strong electric filed guarantees the fast movement of electron. As a results, corona current increase rapidly in a short time from $t_{1}$ to $t_{2}$. The electric field decreases fast with the distance away from bar plate. Ionization caused by electron weakens. On the contrary, the attachment of electron is enhanced.

The decreasing of current right after the peak of the main pulse is due to the electric field reduction in the electrode gap caused by the existence of negative ion cloud and electron clusters which move towards the anode directionally [41]. In order to maintain the balance field by requiring fewer electrons into the anode surface, so from $\mathrm{t}_{4}-\mathrm{t}_{6}$, only a small $\mathrm{DC}$ current $(\mathrm{I}=1 \mathrm{~mA})$ will remain in the circuit.

\subsection{Electron mean energy distribution (EMED)}

Electron-impact reactions (e.g., ionization and excitation) significantly affect the corona discharge. The rate of collision between a molecule/atom and an electron and the transport coefficient of an electron (e.g., electron diffusion coefficient and mobility) are functions of the electron mean energy (EME) [42]. Therefore, research on the electron mean energy distribution (EMED) during a corona discharge pulse cycle is extremely important for a comprehensive understanding of its physical mechanism [42]. Fig. 4 demonstrates the EMED along the axis at 6 typical time point during a pulse cycle, in which " $0 \mathrm{~mm}$ " represents the position of the plate electrode (anode) while " $3.3 \mathrm{~mm}$ " indicates the position of the bar electrode (cathode). After comparing with literature [42], it is interesting to note that EMED has the same development tendency with the electric field distribution (EFD) during a pulse cycle (Fig. 5), because field accelerating is the main way for electrons to gain energy [43]. So, the electric field distortion caused by the directional movement of charged particles in external field plays a key role in EMED, which can explain the phenomena of Fig. 4 as follow. At the beginning of pulse cycle $\left(t_{1}\right)$, the maximum of EME occurs near the cathode, and declines to its minimum near the anode in a form of exponential decay, this is owing to the space shape of bar-plate electrode. As time goes on $\left(t_{2}-t_{6}\right)$, the maximum of EME appears in the field ionization layer, and moves with the field ionization layer from cathode to anode, with its value reduces gradually. In the process of electron avalanche moving to the anode, collision ionization and adsorption reaction create a large number of charged particles. The internal electric field caused by electrode, ion cloud, and electron clusters which directionally move in the external electric field, distorts the electric field between bar and plane electrode. Furthermore, EME has a slightly increase near the anode region $(0-1 \mathrm{~mm})$ with time progresses $\left(t_{1}-t_{6}\right)$. It's for that more and more electrons reach the anode during this period, electric field is strengthened by the internal electric field between anode and electrons near the anode.

\subsection{Electron density distribution (EDD)}

As reported [14, 44], collision ionization between high-energy electrons and neutral molecules are seen as the major chemi-ionization process in the micro-process of corona discharge, and electrons are the main energy transfer carrier between electric field and heavy particles, consequently. Investigation on the EDD can be of

Table 3. The key reactions at different instants

\begin{tabular}{c|c}
\hline Time & The key reactions at different instants \\
\hline$t_{1}$ & $\mathrm{R}_{1}, \mathrm{R}_{2}, \mathrm{R}_{3}, \mathrm{R}_{7} \mathrm{R}_{10}, \mathrm{R}_{22}, \mathrm{R}_{8} \ldots$ \\
\hline$t_{2}$ & $\mathrm{R}_{1}, \mathrm{R}_{22}, \mathrm{R}_{2}, \mathrm{R}_{7}, \mathrm{R}_{10}, \mathrm{R}_{3}, \mathrm{R}_{8} \ldots$ \\
\hline$t_{3}$ & $\mathrm{R}_{1}, \mathrm{R}_{2}, \mathrm{R}_{22}, \mathrm{R}_{7}, \mathrm{R}_{3}, \mathrm{R}_{10}, \mathrm{R}_{8} \ldots$ \\
\hline$t_{4}$ & $\mathrm{R}_{2}, \mathrm{R}_{1}, \mathrm{R}_{22}, \mathrm{R}_{7}, \mathrm{R}_{8}, \mathrm{R}_{10}, \mathrm{R}_{3} \ldots$ \\
\hline$t_{5}$ & $\mathrm{R}_{2}, \mathrm{R}_{1}, \mathrm{R}_{22}, \mathrm{R}_{7}, \mathrm{R}_{8}, \mathrm{R}_{10}, \mathrm{R}_{3} \ldots$ \\
\hline$t_{6}$ & $\mathrm{R}_{1}, \mathrm{R}_{22}, \mathrm{R}_{2}, \mathrm{R}_{7}, \mathrm{R}_{8}, \mathrm{R}_{10}, \mathrm{R}_{3} \ldots$ \\
\hline
\end{tabular}




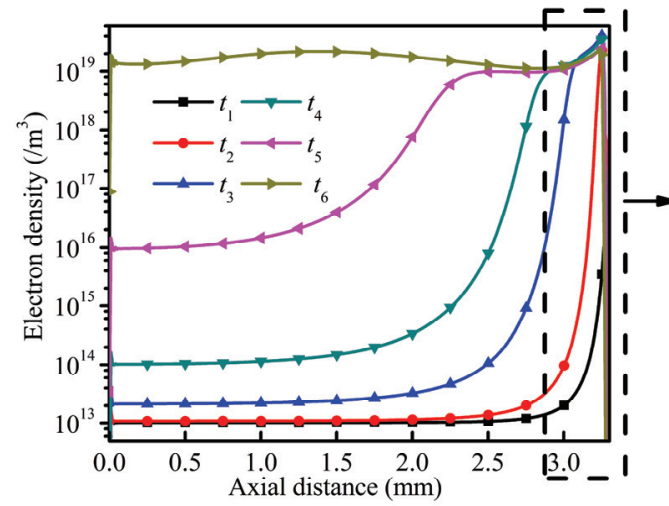

(a)

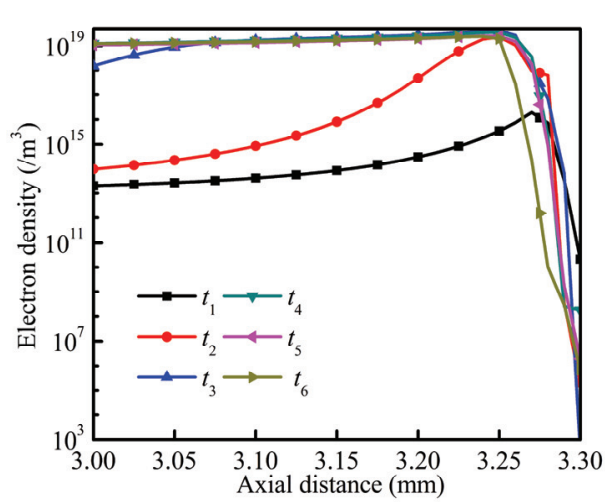

(b)

Fig. 6. EDD along the axis: (a) EDD along the whole axis; (b) EDD near the cathode

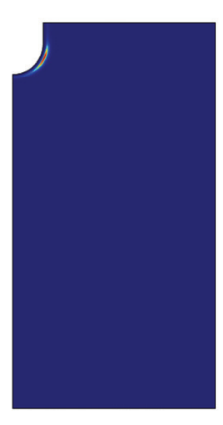

$t_{1}$

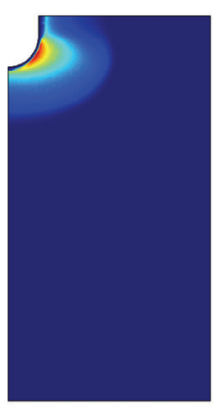

$t_{4}$
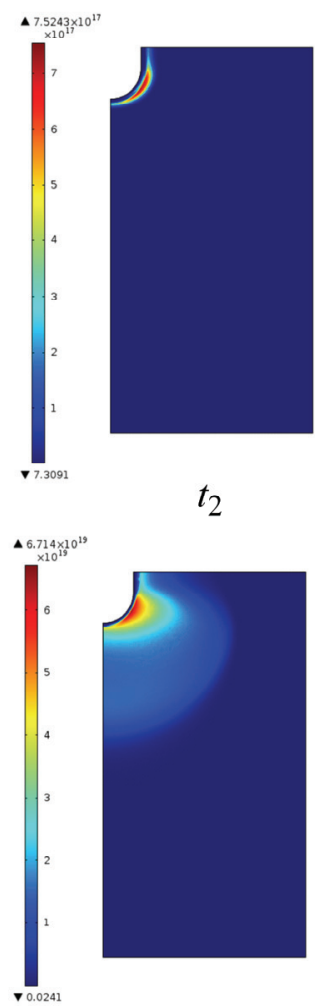

$t_{5}$
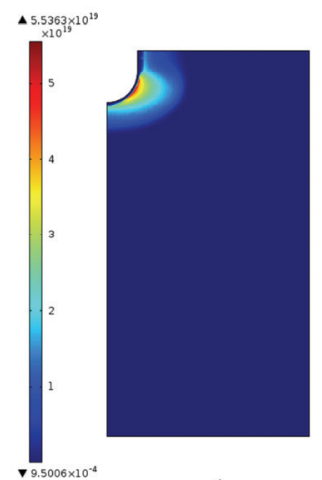

$t_{3}$

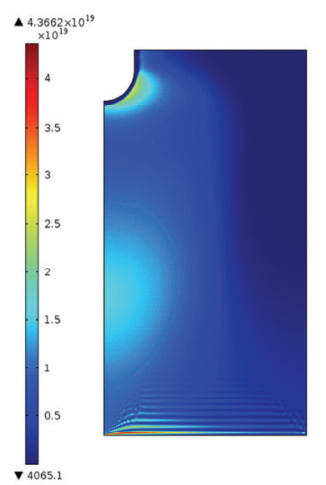

$t_{6}$
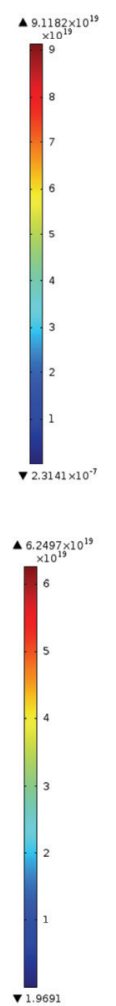

Fig. 7. 2D-EDD at 6 typical time

important value to understand the physical mechanism of air discharge. Fig. 6(b) shows the thickness of cathode sheath is about $30 \mu \mathrm{m}$, and the electron density (ED) keeps almost 0 in the cathode sheath in the whole discharge process, because of the exclusion which prevents electrons to enter into the cathode. The maximum of ED appears in the outer layer of cathode sheath where the field ionization effect is strongest due to the concentrated electric field. As time increases $\left(t_{1}-t_{3}\right)$, the ED in the outer layer of cathode sheath rapidly increase to the maximum. Then the ED begin to decrease to a stable point until the time point $t_{5}$. Fig. 6(a) shows the EDD along the whole axis at 6 typical time during a pulse cycle. As time increases $\left(t_{1}-t_{6}\right)$, the EDD moves towards the anode, and the density of electrons increases gradually. Fig. 7 shows the development tendency of EDD clearly. We also noticed that ED has an abnormal increase near the anode at the end of pulse cycle $\left(\mathrm{t}_{6}\right)$. The reasons are as follows. Firstly, the secondary electron emission caused by the collision between negative ions and anode. Secondly, more and more positive ions gain electrons from the anode in order to restore electrical neutrality. Lots of light radiation phenomena usually are accompanied with air discharge process, whose intensity generally depends on the energy transfer intensity caused by collision between electrons and other particles, therefore, the EDD is often related to 


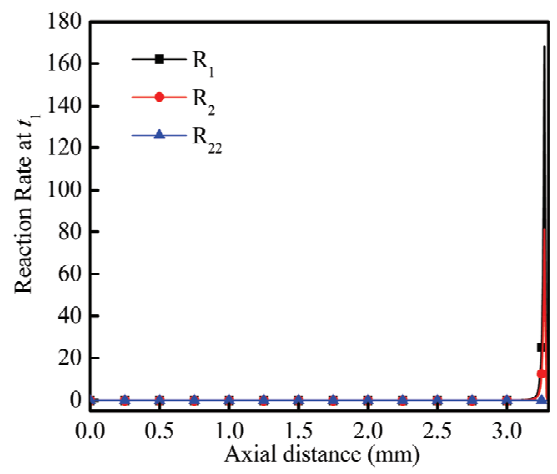

(a)

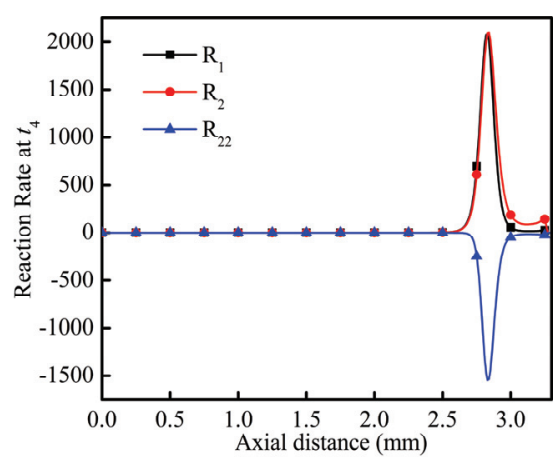

(d)

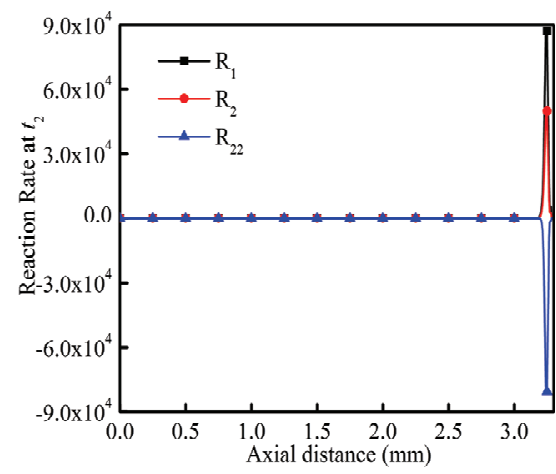

(b)

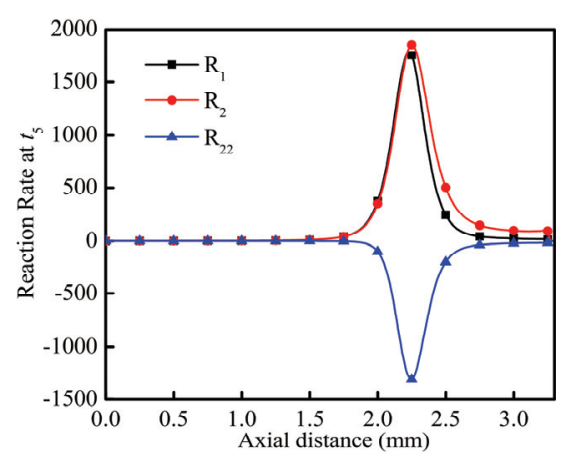

(e)

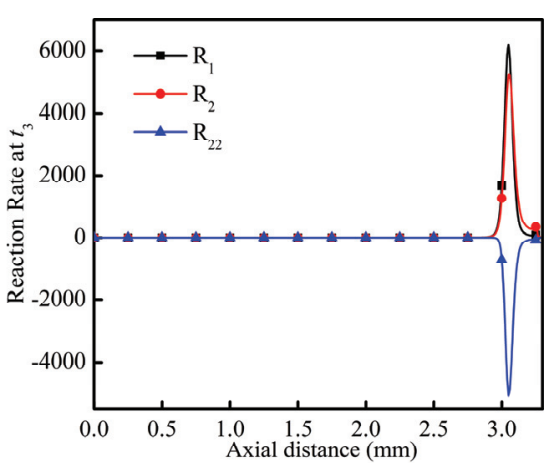

(c)

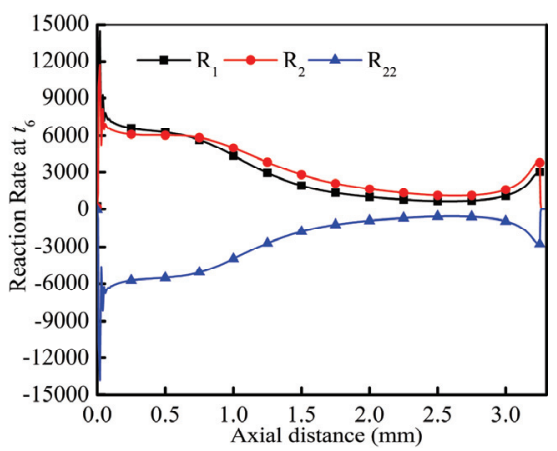

(f)

Fig. 8. Top 3 reaction rate at 6 typical time, (a) $t_{1}$, (b) $t_{2}$, (c) $t_{3}$, (d) $t_{4}$, (e) $t_{5}$, (f) $t_{6}$ (Note: two-body reaction coefficient unit is $\mathrm{m}^{3} \mathrm{~s}^{-1}$; three-body reaction coefficient unit of $\mathrm{m}^{6} \mathrm{~s}^{-1}$.)

corona imaging. This is why the 2D-EDD at t6 has a very similar distribution with the images of corona discharge in literature [45].

\subsection{Generation and dissipation performances of electron}

The rate of collision reaction can reflect the strength of that reaction process in air discharge. According to the existing literature, simplified chemical model which contains limited charged particles and collision reactions is considered into corona discharge process. Consequently, behavior of charged particles and chemical reactions in micro-process of corona discharge are still unclear. In this paper, 7 reactions whose maximum rate along the axis ranked top 7 at 6 representative time points are listed in Tab. 3. From Tab. 3 we note that, $R_{1}, R_{2}$ and $R_{22}$ play the main role in the whole discharge process. $R_{1}$ and $R_{2}$ are collision reactions of electrons collision reactions associated with $\mathrm{N}_{2}$ and $\mathrm{O}_{2}$ respectively. Meanwhile, they are the main process of electron proliferation. Besides, reaction rates of $R_{1}$ and $R_{2}$ almost show the same curve (Fig. 8). $R_{22}$ is a recombination reaction of $\mathrm{N}_{2}, \mathrm{~N}_{2}^{+}$and $e$, which play a dominant role in the dissipation process of electron. Fig. 8 indicates that generation and dissipation reactions of electron are mainly concentrated in field ionization layer because of the high electric field. With the increase of time $\left(t_{1}-t_{2}\right)$, the rates of $R_{1}, R_{2}$ and $R_{22}$ rapidly increase from a very limited value to the maximum. A large number of electrons and ions are produced in this process which would lead to a fast rising front in the discharge current. Sequentially, from $t_{2}-t_{5}$, the rates of $R_{1}, R_{2}$ and $R_{22}$ begin to fall slowly due to the declining electric field in field ionization layer. At the end of pulse cycle $\left(t_{6}\right)$, the rates of $\mathrm{R}_{1}, \mathrm{R}_{2}$ and $\mathrm{R}_{22}$ increase with an exception state, because at this time, the electrode gap restores to electrical neutrality, so ions and electrons are widely distributed in the whole gap, so the electric field is extremely distorted.

\section{Conclusions}

In this paper, a plasma chemical model for simulation of bar-plate DC negative corona discharge under atmospheric air is presented. Under the condition of applied voltage of $-5.0 \mathrm{kV}$, microcosmic characteristics at 6 typical time points during a pulse period are used to describe the characteristics of electrons of negative corona discharge in air. The important conclusions based on the current investigations are as follows:

1) At the beginning of the pulse cycle, the maximum of electron mean energy (EME) occurs near the cathode, and declines to its minimum near the anode in a form of exponential decay. As time goes on, the maximum of EME appears in the field ionization layer, and moves along the field ionization layer from cathode to anode, 
meanwhile its value reduces gradually.

2) Within a pulse process, the electron density (ED) in cathode sheath almost keeps almost 0 , and the maximum of ED appears in the outer layer of the cathode sheath. The electron density distribution (EDD) moves towards the anode, and the density of space gap increases gradually.

3) $R_{1}, R_{2}$ and $R_{22}$ play the main role in the whole discharge process. $R_{1}$ and $R_{2}$ which are the collision reaction of electrons associated respectively with $\mathrm{N}_{2}$ and $\mathrm{O}_{2}$ regarded as the main process of electron proliferation, besides, reaction rates of $R_{1}$ and $R_{2}$ almost keep the same curve. $R_{22}$ is the recombination reaction of $\mathrm{N}_{2}, \mathrm{~N}_{2}{ }^{+}$and $\mathrm{e}$, which plays a dominant role in the dissipation process of electrons.

\section{Acknowledgements}

This work was supported by the National Basic Research Program of China (Grant Nos. 2011CB209401).

\section{References}

[1] H. Yin, J.L. He, B. Zhang, "Finite volume-based approach for the hybrid ion-flow field of UHVAC and UHVDC transmission lines in parallel", IEEE Transactions on Power Delivery, vol. 26, no. 4, pp. 2809-2820, September 2005.

[2] L. Chen, X. Bian, L. Wang, "Effect of rain drops on corona discharge in alternating current transmission lines with a corona cage", Japanese Journal of Applied Physics, vol. 51, no. 9S2, pp. 09MG02, September 2012.

[3] X.B. Bian, L.C. Chen, D. Yu, "Impact of surface roughness on corona discharge for 30-year operating conductors in $500-\mathrm{kV}$ ac power transmission line", IEEE Transactions on Power Delivery, vol. 27, no. 3, pp. 1693-1695, July 2012.

[4] Y. Kim, K. Shong, "The characteristics of UV strength according to corona discharge from polymer insulators using a UV sensor and optic lens", IEEE Transactions on Power Delivery, vol. 26, no. 3, pp. 1579-1584, July 2011.

[5] G. Horvath, M. Zahoran, N. J. Mason, "Methane decomposition leading to deposit formation in a DC positive CH4-N2 corona discharge", Plasma Chemistry and Plasma Processing, vol. 31, no. 2, pp. 327-335, April 2011.

[6] C. Labay, C. Canal, M.J. García-Celma, "Influence of corona plasma treatment on polypropylene and polyamide 6.6 on the release of a Model Drug", Plasma Chemistry and Plasma Processing, vol. 30, no. 6, pp. 327-335, December 2010.

[7] M. Redolfi, N. Aggadi, X. Duten, "Oxidation of acetylene in atmospheric pressure pulsed corona discharge cell working in the nanosecond regime", Plasma Chemistry and Plasma Processing, vol. 29, no. 3, pp. 173-195, June 2009.

[8] B. M. Penetrante, J. N. Bardsley, M. C. Hsiao, "Kinetic analysis of non-thermal plasmas used for pollution control", Japanese journal of applied physics, vol. 36, no. 7S, pp. 5007, July 1997.

[9] S. Masuda, H. Nakao, "Control of NOx by positive and negative pulsed corona discharges", IEEE Transactions on Industry Applications, vol. 26, no. 2, pp. 374-383, April 1990.

[10] W. Peukert, C. Wadenpohl, "Industrial separation of fine particles with difficult dust properties", Powder Technology, vol. 118, no. 1, pp. 136-148, August 2001.

[11] C. Lanzerstorfer, "Solid/Liquid-Gas Separation with Wet Scrubbers and Wet Electrostatic Precipitators: A Review", Filtration and Separation, vol. 37, no. 5, pp. 30-34, June 2000.

[12] G. Deli, Y. Xuechang, Z. Fei, et al, "Experimental study on indoor air cleaning technique of nano-titania catalysis under plasma discharge", Plasma Science and Technology, vol. 10, no. 2, pp. 216, April 2008.

[13] I. Fofana, A. Beroual, "A model for long air gap discharge using an equivalent electrical network", IEEE Transactions on Dielectrics and Electrical Insulation, vol. 3, no. 2, pp. 273-282, April 1996.

[14] S. Nijdam, K. Miermans, E. M. van Veldhuizen, “A peculiar streamer morphology created by a complex voltage pulse", IEEE Transactions on Plasma Science, vol. 39, no. 11, pp. 2216-2217, November 2011.

[15] K. Sekimoto, M. Takayama, "Influence of needle voltage on the formation of negative core ions using atmospheric pressure corona discharge in air", International Journal of Mass Spectrometry, vol. 261, no. 1, pp. 38-44, March 2007.

[16] T. J. Sommerer, M. J. Kushner, "Numerical investigation of the kinetics and chemistry of $\mathrm{rf}$ glow discharge plasmas sustained in $\mathrm{He}, \mathrm{N}_{2}, \mathrm{O}_{2}, \mathrm{He} / \mathrm{N}_{2} / \mathrm{O}_{2}$, $\mathrm{He} / \mathrm{CF}_{4} / \mathrm{O}_{2}$, and $\mathrm{SiH} 4 / \mathrm{NH} 3$ using a Monte Carlofluid hybrid model", Journal of applied physics, vol. 761, no. 4, pp. 1654-1673, March 1992.

[17] J. Nahomy, C. M. Ferreira, B. Gordiets, "Experimental and theoretical investigation of a $\mathrm{N}_{2}-\mathrm{O}_{2}$ DC flowing glow discharge", Journal of Physics D: Applied Physics, vol. 28, no. 4, pp. 738, April 1995.

[18] S. Pancheshnyi, M. Nudnova, A. Starikovskii, "Development of a cathode-directed streamer discharge in air at different pressures: experiment and comparison with direct numerical simulation", Physical Review E, vol. 71, no. 1, pp. 016407, January 2005.

[19] X. H. Liu, W. He, F. Yang, " Numerical simulation and experimental validation of a direct current air corona discharge under atmospheric pressure", Chinese Physics B, vol. 21, no. 7, pp. 075201, July 2012.

[20] W. He, X. H. Liu, F. Yang, "Numerical simulation of 
direct current glow discharge in air with experimental validation", Japanese Journal of Applied Physics, vol. 51, no. 2R, pp. 026001, February 2012.

[21] D. S. Antao, D. A. Staack, A. Fridman, "Atmospheric pressure dc corona discharges: operating regimes and potential applications", Plasma Sources Science and Technology, vol. 18, no. 3, pp. 035016, August 2009.

[22] F. F. Wu, R. J. Liao, K. Wang, "Numerical Simulation of the Characteristics of Heavy Particles in Bar-Plate DC Positive Corona Discharge Based on a Hybrid Model", IEEE Transactions on Plasma Science, vol. 42, no. 3, pp. 868-878, March 2014.

[23] G. E. Georghiou, A. P. Papadakis, R. Morrow, "Numerical modelling of atmospheric pressure gas discharges leading to plasma production", Journal of Physics D: Applied Physics, vol. 38, no. 20, pp. R303, October 2005.

[24] C. Li, U. Ebert, W. Hundsdorfer, "Spatially hybrid computations for streamer discharges with generic features of pulled fronts: I. Planar fronts", Journal of Computational Physics, vol. 229, no. 1, pp. 200-220, January 2010.

[25] T. N. Tran, I. O. Golosnoy, P. L. Lewin, "Numerical modelling of negative discharges in air with experimental validation", Journal of Physics D: Applied Physics, vol. 44, no. 1, pp. 015203, January 2011.

[26] B. F. Gordiets, C. M. Ferreira, V. L. Guerra V L, "Kinetic model of a low-pressure $\mathrm{N}_{2}-\mathrm{O}_{2}$ flowing glow discharge", IEEE Transactions on Plasma Science, vol. 23, no. 4, pp. 750-768, August 1995.

[27] S. Mahadevan, L. L. Raja, "Simulations of directcurrent air glow discharge at pressures $\sim 1$ Torr: Discharge model validation", Journal of Applied Physics, vol. 107, no. 9, pp. 093304, May 2010.

[28] A. A. Kulikovsky, "The role of photoionization in positive streamer dynamics", Journal of Physics D: Applied Physics, vol. 33, no. 12, pp. 1514, June 2000.

[29] N. Liu, V. P. Pasko, "Effects of photoionization on similarity properties of streamers at various pressures in air", Journal of Physics D: Applied Physics, vol. 39, no. 2, pp. 327, January 2006.

[30] S. Nijdam, F. Van De Wetering, R. Blanc, "Probing photo-ionization: experiments on positive streamers in pure gases and mixtures", Journal of Physics D: Applied Physics, vol. 43, no. 14, pp. 14520, April 2010.

[31] M. B. Zhelezniak, A. K. Mnatsakanian, S. V. Sizykh, "Photoionization of nitrogen and oxygen mixtures by radiation from a gas discharge", High Temperature Science, vol. 20, no. 3, pp. 423-428, November 1982.

[32] I. A. Kossyi, A. Y. Kostinsky, A. A. Matveyev, "Kinetic scheme of the non-equilibrium discharge in nitrogen-oxygen mixtures", Plasma Sources Science and Technology, vol. 1, no. 3, pp. 207, August 1992.

[33] P. Ségur, A. Bourdon, E. Marode, "The use of an improved Eddington approximation to facilitate the calculation of photoionization in streamer discharges", Plasma Sources Science and Technology, vol. 15, no. 4, pp. 648, November 2006.

[34] T. Farouk, B. Farouk, D. Staack, "Simulation of dc atmospheric pressure argon micro glow-discharge", Plasma Sources Science and Technology, vol. 15, no. 4, pp. 676, November 2006.

[35] G. J. M. Hagelaar, F. J. De Hoog, G. M. W. Kroesen, "Boundary conditions in fluid models of gas discharges", Physical Review E, vol. 62, no. 1, pp. 1452, July 2000.

[36] Y. Gosho, "Enhancement of dc positive streamer corona in a point-plane gap in air due to addition of a small amount of an electronegative gas", Journal of Physics D: Applied Physics, vol. 14, no. 11, pp. 2035, November 1981.

[37] J. Y. Won, P. F. Williams, "Experimental study of streamers in pure $\mathrm{N}_{2}$ and $\mathrm{N}_{2} / \mathrm{O}_{2}$ mixtures and $\mathrm{a} \approx 13$ cm gap", Journal of Physics D: Applied Physics, vol. 35, no. 3, pp. 205, February 2002.

[38] W. X. Sima, Q. J. P, Q. Yang, "Local electron mean energy profile of positive primary streamer discharge with pin-plate electrodes in oxygen - nitrogen mixtures", Chinese Physics B, vol. 22, no. 1, pp. 015203, January 2013.

[39] R. Zentner, "Rise Time of Negative Corona Pulses", Zeitschrift Fur Angewandte Physik, vol. 29, no. 5, pp. 294-\&, January 1970.

[40] C. Soria-Hoyo, F. Pontiga, A. Castellanos, "Particlein-cell simulation of Trichel pulses in pure oxygen", Journal of Physics D: Applied Physics, vol. 40, no. 15, pp. 4552, August 2007.

[41] R. J. Liao, F. F. Wu, L.J. Yang, "Investigation on Microcosmic Characteristics of Trichel Pulse in BarPlate DC Negative Corona Discharge Based on a Novel Simulation Model", International Review of Electrical Engineering, vol. 8, no. 1, 2013.

[42] W.X. Sima, Q.J. Peng, Q. Yang, "Local electron mean energy profile of positive primary streamer discharge with pin-plate electrodes in oxygen - nitrogen mixtures", Chinese Physics B, vol. 22, no. 1, pp. 015203, January 2013.

[43] J. D. Bourke, C. T. Chantler, "Electron energy loss spectra and overestimation of inelastic mean free paths in many-pole models", The Journal of Physical Chemistry A, vol. 116, no. 12, pp. 3202-3205, March 2012.

[44] J. Chen, J. H. Davidson, "Ozone production in the positive DC corona discharge: Model and comparison to experiments", Plasma chemistry and plasma processing, vol. 22, no. 4, pp. 495-522, December 2002.

[45] D. Staack, B. Farouk, A. Gutsol, "Characterization of a dc atmospheric pressure normal glow discharge", Plasma Sources Science and Technology, vol. 14, no. 4, pp. 700, November 2005. 


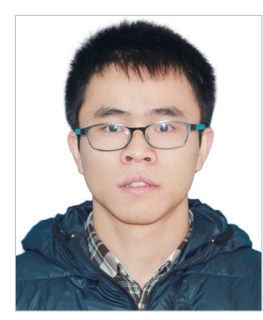

Kang-Lin Liu He was born in Chongqing, China in 1991.He received B.S degree in electrical engineering from Chongqing University. He is currently a Ph.D. candidate in the school of Electrical Engineering, Chongqing University, China. His research interests are corona discharge and the measurement of space charge in air.

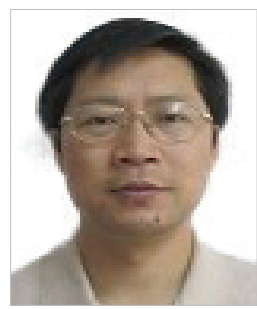

Rui-jin Liao He was born in Sichuan, China in 1963. He received the M.S. and Ph.D. degrees in electrical engineering from Xi'an Jiaotong University, China and Chongqing University, China, respectively. Since 1999 he is a professor of Electrical Engineering College at Chongqing University, China. His research activities lie in the field of on-line monitoring of insulation condition and fault diagnosis for high voltage apparatus, as well as ageing mechanism and diagnosis for power transformer. He is author / coauthor of one book and over 90 journal and international conferences.

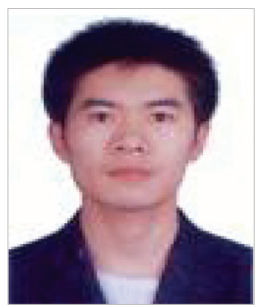

Xue-Tong Zhao was born in Henan, China in 1984. He received the B.S in College of Electrical Engineering from Henan Polytechnic University. Currently, he is a graduate student of high voltage and insulation technology in Xi'an Jiaotong University. His main research field is insulation material and zinc oxide varistor ageing. 